\title{
The effectiveness of afatinib and osimertinib in a Chinese patient with advanced lung adenocarcinoma harboring a rare triple EGFR mutation (R670W/H835L/L833V): a case report and literature review
}

This article was published in the following Dove Press journal: OncoTargets and Therapy

\author{
Bao-Dong Qin* \\ Xiao-Dong Jiao* \\ Ling-Yan Yuan* \\ Ke Liu \\ Zhan Wang \\ Wen-Xing Qin \\ Yuan-Sheng Zang
}

Department of Medical Oncology, Changzheng Hospital, Naval Medical University, Shanghai 200072, China

*These authors contributed equally to this work
Correspondence: Yuan-Sheng Zang Department of Medical Oncology, Changzheng Hospital, Naval Medical University, 64 Hetian Road, Shanghai, 200072, China

Tel/fax +86 2I 66540109 ext 8039 Email doctorzangys@।63.com

\begin{abstract}
In patients without tissue availability at presentation, the analysis of cell-free DNA derived from liquid biopsy samples, in particular from plasma, represents an established alternative for providing epidermal growth factor receptor (EGFR) mutational testing for treatment decision-making. Compared with quantitative polymerase chain reaction and digital polymerase chain reaction-targeted methods, next-generation sequencing can provide more information about tumor molecular alterations, especially EGFR mutations. Here, we present a case of a patient with non-small cell lung cancer (NSCLC) harboring 3 uncommon mutations of EGFR-R670W in exon 17 and $\mathrm{H} 833 \mathrm{~V}$, and $\mathrm{H} 835 \mathrm{~L}$ in exon 21, as shown by next-generation sequencing of plasma cell-free DNA. To the best of our knowledge, this is the first case report of a patient harboring the R670W mutation. The patient responded well to second-generation tyrosine kinase inhibitors (TKIs). T790M is an acquired resistant mutation in patients with R670W, H833V, and H835L. This is also the first case of a patient harboring the H833V/H835L/T790M triple mutation; the patient had a good response to the third-generation TKI osimertinib. In this work, we also performed a literature review on the clinical characteristics of NSCLC patients with the H833V/ H835L double mutation, together with a descriptive analysis about their response to EGFR TKI monotherapy as a first-line treatment, according to data from previous case reports. The results showed that the cohort of NSCLC patients with H833V/H835L responded well to EGFR TKIs; thus, before treatment in clinical practice, screening for EGFR mutations should be conducted and EGFR TKIs should be preferred in NSCLC patients with H833V/H835L mutations.
\end{abstract}

Keywords: non-small cell lung cancer, cell-free DNA, EGFR mutation, afatinib, osimertinib

\section{Introduction}

Mutations in epidermal growth factor receptor (EGFR) are the most common drivers of non-small cell lung cancer (NSCLC) growth, with an incidence of $\sim 10 \%-15 \%$ in Caucasian and $40 \%$ in Asian patients. ${ }^{1}$ The 2 most common EGFR mutations in lung cancer are the exon 19 deletion and the L858R mutation in exon 21, which account for about $90 \%$ of all mutations in this gene. ${ }^{2}$ To date, several large Phase III clinical trials have demonstrated that NSCLC patients with classical/sensitizing EGFR mutations responded well to EGFR tyrosine kinase inhibitors (TKIs) as a standard first-line treatment. The remaining $10 \%$ of EGFR mutations, so-called uncommon mutations, are a heterogeneous group of genomic alterations that occur within exons 18-21. 
Recently, the most reported uncommon EGFR mutations have included G719X (where X indicates A, S, C, or D) in exon 18, L861Q in exon 20, and S768I in exon 21. According to a recent molecular epidemiological study in Asia, these 3 mutations constitute $\sim 6 \%$ of all EGFR mutations. ${ }^{3}$ The effects of EGFR TKIs in patients with uncommon EGFR mutations remain largely unknown. A previous study found that first-generation EGFR-TKIs are active in patients with these uncommon mutations, but are less effective than in patients with common mutations. ${ }^{4}$ The combined post hoc analysis of Lux-Lung 2/3/6 led to the same conclusion that afatinib was active in NSCLC tumors that harbored certain types of uncommon EGFR mutations, especially G719X, L861G, and S768I, but was less active in tumors with other mutations. ${ }^{5}$

Currently, with the advances in detection techniques and precise detection assays, especially next-generation sequencing (NGS), an increasing number of rare or atypical EGFR mutations have been identified, and multiple EGFR mutations have been detected in a growing number of NSCLC patients. However, the effectiveness of treatment in patients with these rare mutations are unknown, as is the optimal treatment for patients with multiple rare EGFR mutations. A previous study demonstrated that the combination of a rare mutation and common one or between a rare mutation and another rare one with sensitizing effects, was associated with an intermediate outcome between the 2 singular situations. In general, the association of a sensitizing mutation seems to attenuate the negative effect of the rare alteration, so that the prognosis shifts toward the progression-free survival (PFS) and overall survival observed with the common mutation. ${ }^{6}$ However, the combination of a rarer mutation and another rarer one, or even a triple rarer mutation needs further study.

Here, we present a case of an NSCLC patient harboring 3 uncommon mutations, namely, EGFR-R670W in exon 17, $\mathrm{H} 833 \mathrm{~V}$ in exon 21, and H835L in exon 21, who was treated with afatinib and osimertinib. We also performed a literature review on the clinical characteristics of NSCLC patients with the $\mathrm{H} 833 \mathrm{~V} / \mathrm{H} 835 \mathrm{~L}$ double mutation, together with a descriptive analysis about their response to EGFR TKI monotherapy as first-line treatment, according to data from previous case reports.

\section{Case report}

A 36-year-old male, nonsmoker, presented to our hospital with a 1-month history of back pain. MRI revealed multiple lesions in lumbar (L4) and sacral vertebrae (S2-S3), and a L4 vertebral pathological fracture. A CT scan revealed 2 masses in the superior and lower lobe of the left lung (TNM classification stage 4 [T1N0M1], pleural and bone metastases, Performance Status PS1). Due to the lesions in the spine, the patient underwent surgery. H\&E staining showed morphology typical of adenocarcinoma cells (Figure 1A). Immunohistochemistry demonstrated strong positivity for CK7, and weak staining of TTF-1, Napsin A (Figure 1B-D). Formalin-fixed paraffin-embedded specimens from metastatic specimen were subjected to amplification refractory mutation system (ARMS)-polymerase chain

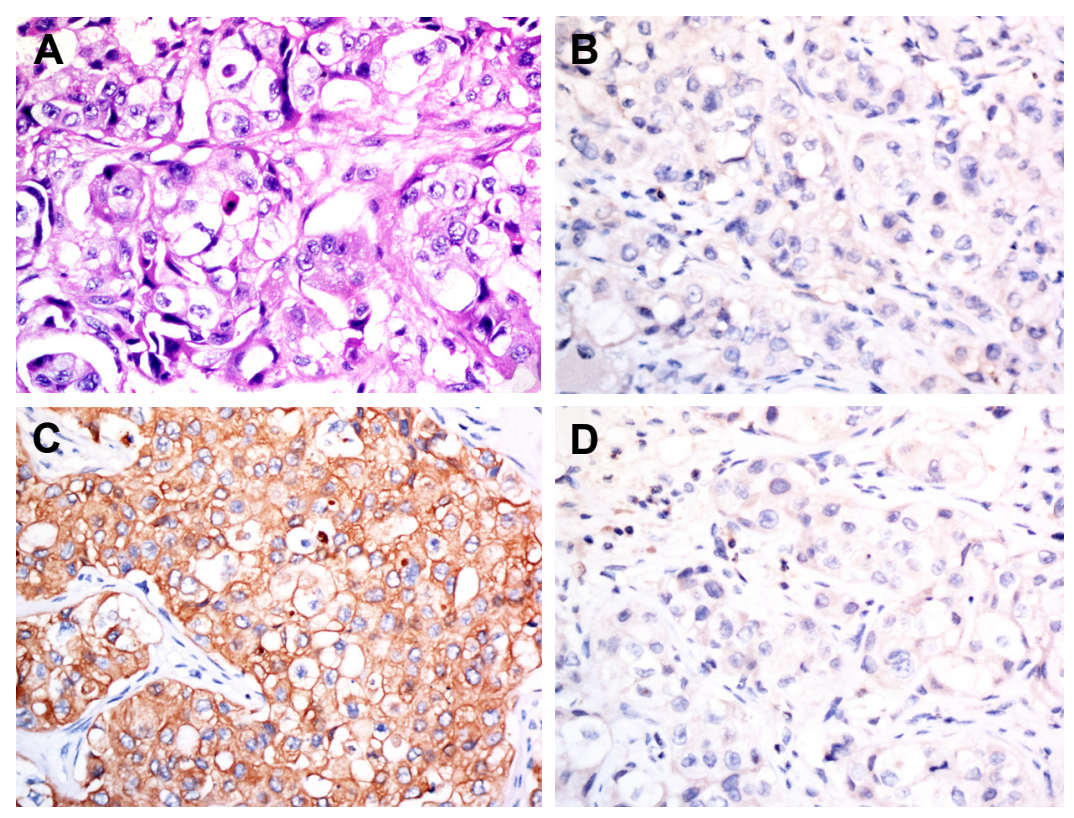

Figure I H\&E staining (A), I00×, and immunohistochemistry (B) for TTF-I, (C) for CK7, (D) for Napsin A, I00×, of the specimen, which showed lung adenocarcinoma. 
reaction (PCR) for common EGFR mutations, which showed no 19del or L858R mutations, and were also negative for anaplastic lymphoma kinase and proto-oncogene tyrosineprotein kinase ROS-1 fusions. However, bone metastasis specimens were decalcified with decalcifying solutions. To exclude the potential influence of decalcifying solution on tests for genetic alterations, NGS of plasma cell-free DNA (cfDNA) was performed. Capture-based ultra-deep targeted sequencing was performed on cfDNA by a panel consisting of all exons and critical introns of 295 cancer-related genes, spanning 2.02 MB of human genome using the HiSequation 4,000 system (Illumina, San Diego, CA, USA). The results showed that the patient had 3 uncommon EGFR mutations: EGFR-R670W in exon 17, H833V in exon 21, and H835L in exon 21 (Figure 2). The patient was treated with oral gefitinib (250 mg, q.d.) on September 25, 2016 (Figure 3). After 1 month, chest CT scans revealed a significant increase in tumor size. According to the response evaluation criteria in solid tumors (version 1.1), the patient was considered to have progressive disease on gefitinib and was thus resistant to gefitinib. Next, we chose pemetrexed and cisplatin as second-line therapy. After 2 cycles, chest CT scan showed a significant decrease in tumor size, indicating a partial response to chemotherapy. However, the patient
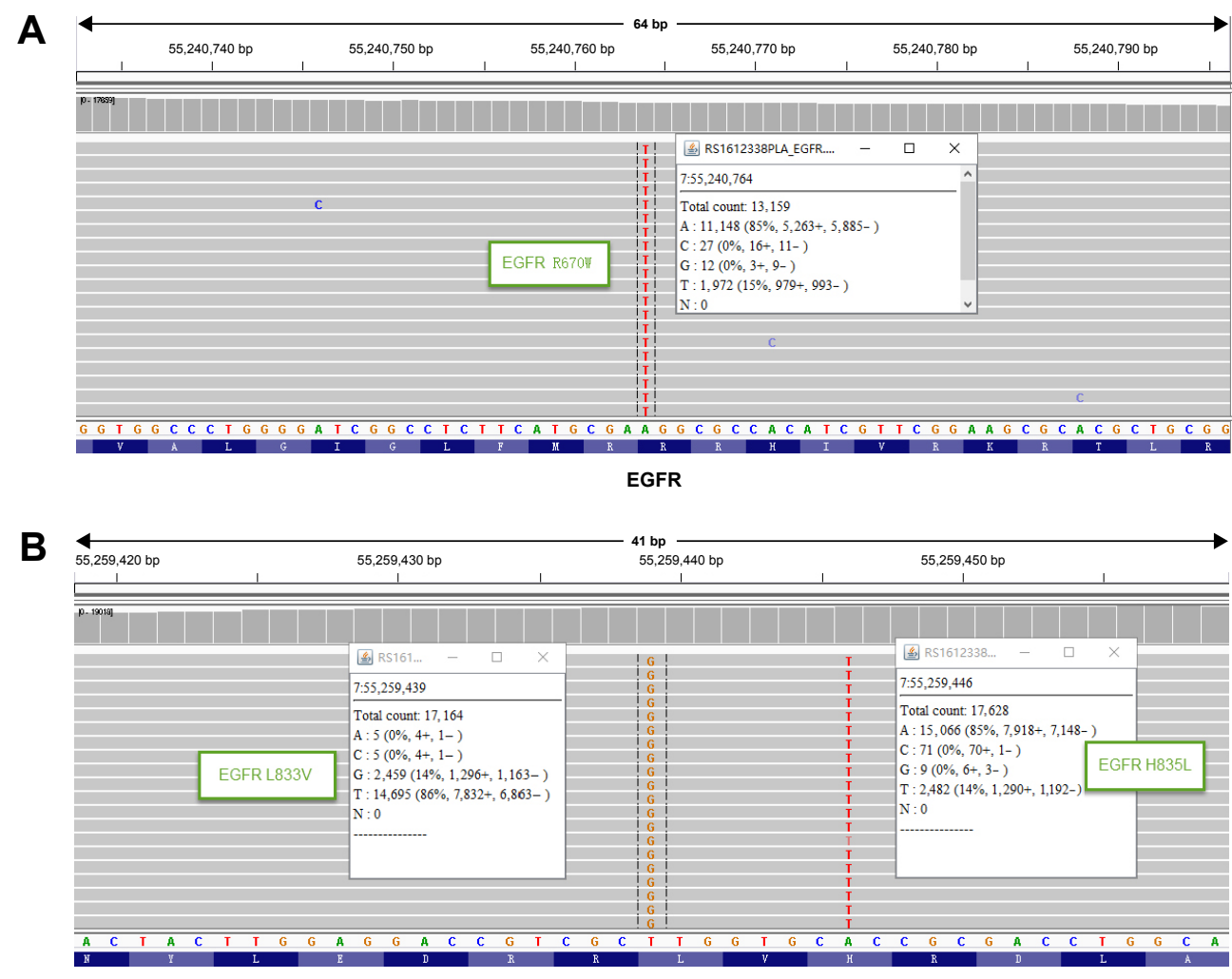

EGFR

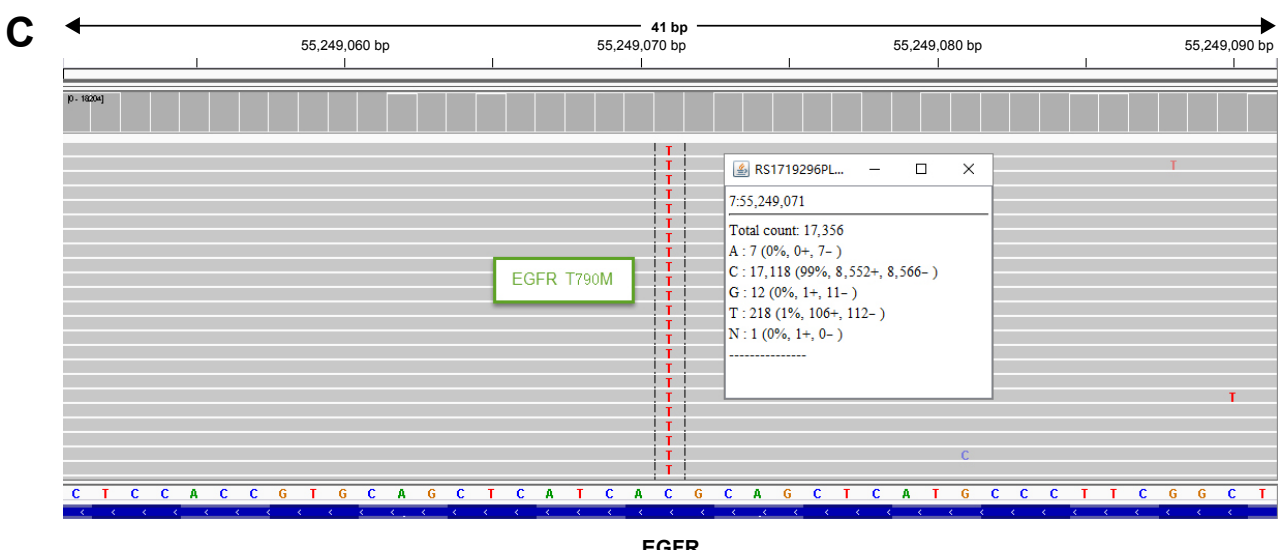

Figure 2 Next-generation sequencing showed R670W in epidermal growth factor receptor exon 17 (A), and H835L, L833V (B), and T790M (C) in exon 21 . 


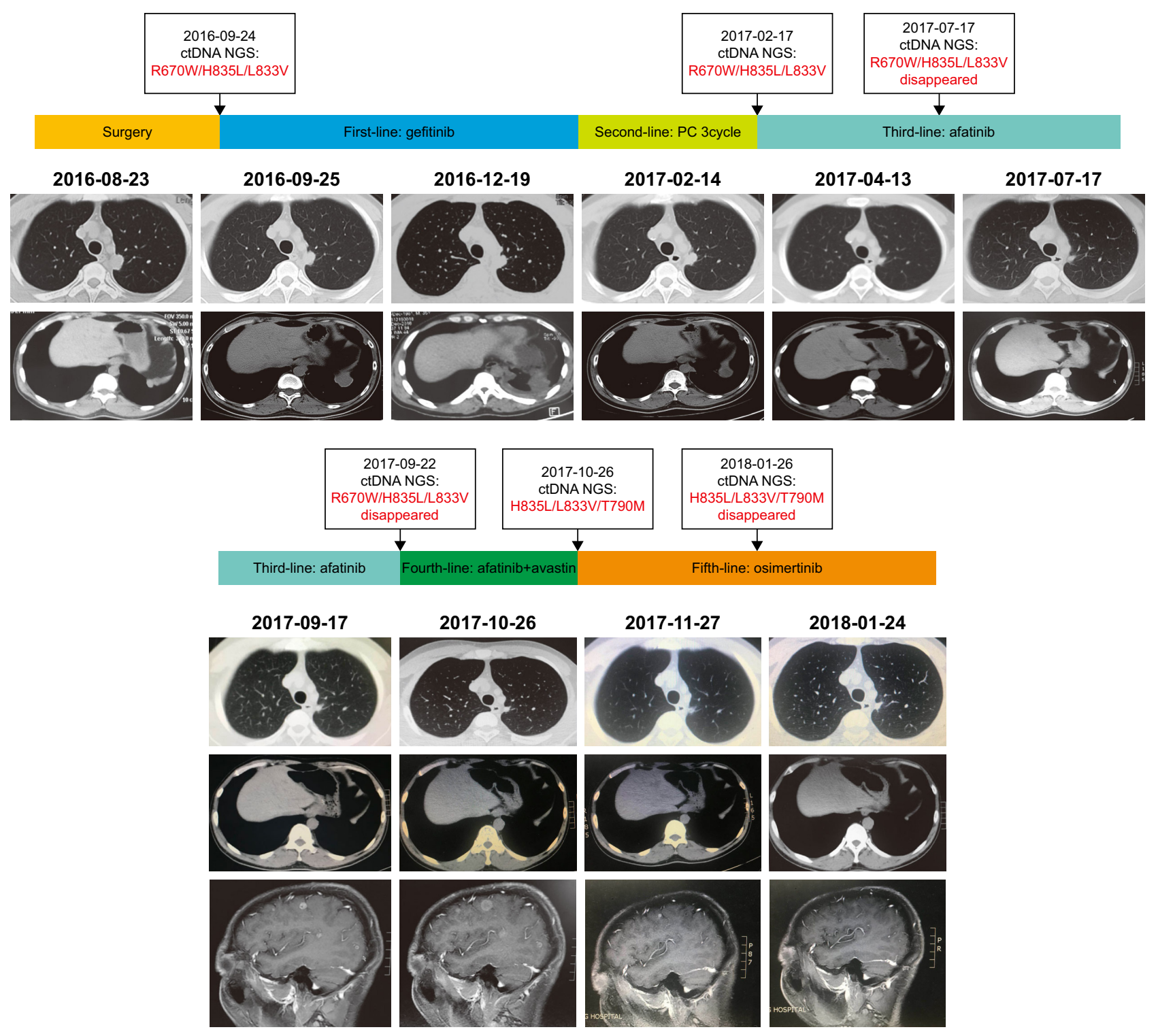

Figure 3 Treatment of lung adenocarcinoma using different regimens, and the results of monitoring the NGS of cfDNA. Abbreviations: NGS, next-generation sequencing; cfDNA, cell-free DNA.

discontinued chemotherapy due to unbearable nausea and vomiting. After adequate communication within our team and with the patient, afatinib was recommended as third-line therapy. After 1 month, the chest $\mathrm{CT}$ scan demonstrated a significant decrease in tumor size, demonstrating that the patient had a partial response to afatinib. On July 17, 2017, NGS of plasma cfDNA showed disappearance of all 3 uncommon EGFR mutations, and by September 17, 2017, the lung lesion was stable. PFS on afatinib was $>7$ months. The most recent brain MRI demonstrated multiple parenchymal brain metastases, but R670W, H835L, and L833V disappeared according to NGS of the plasma. In addition, the T790M mutation was not observed in circulating tumor
DNA by NGS. Afatinib and avastin were subsequently used as fourth-line therapies, but the brain metastatic lesion enlarged after 1 month. On October 26, 2017, NGS of plasma detected resistant EGFR T790M and 2 uncommon mutations, L833V and H835L. Thus, osimertinib, a third-generation TKI, was recommended as fifth-line therapy on October 28, 2017. After 1 month, MRI showed that the lesion was significantly smaller. The last follow-up was on January 24, 2018, at which point both the primary and metastatic brain lesions were stable, and T790M, H835L, and L833V disappeared (Figure 3). Written informed consent has been provided by the patient to have the case details and any accompanying images published. 


\section{Discussion}

We showed the clinical efficacy of afatinib and osimertinib in a patient with NSCLC positive uncommon triple EGFR mutations. This case had several unusual features: three rare EGFR mutations (L833V, H835L, and R670W) were present in the same patient; the first case study of the R670W mutation; T790M was a secondary resistant mechanism in NSCLC patient harboring L833V, H835L, and R670W treated with afatinib; and patient with L833V, H835L, and T790M had a good response to osimertinib.

Complex mutations of L833V and H835L in EGFR rarely coexist in lung cancer, and few published studies have detailed the clinical profiles of this subset of lung cancer patients. To the best of our knowledge, only 10 patients with complex mutations of $\mathrm{H} 833 \mathrm{~V}$ and $\mathrm{H} 835 \mathrm{~L}$ have been reported, including our patient. A total of 6 patients had relevant clinical characteristics reported (Table 1). Most reported cases only had a double mutation of $\mathrm{H} 833 \mathrm{~V}$ and $\mathrm{H} 835 \mathrm{~L}$, whereas this study and one by Frega reported a patient with NSCLC harboring a rare triple EGFR mutation. ${ }^{7}$ Our patient is the first Chinese patient to have this uncommon triple EGFR mutation (L833V, H835L, and R670W). According to a previous study, EGFR H835L mutations are always combined with L833V mutations; there has only been 1 report of a patient with the L833V mutation combined with the G719S mutation. ${ }^{8}$ Therefore, we speculated that the L833V mutation may be accompanied by other mutations, and L833V-H835L complex mutations may be unique mutations in NSCLC. All 6 patients shown in Table 1 had adenocarcinoma with complex mutations of $\mathrm{H} 833 \mathrm{~V}$ and $\mathrm{H} 835 \mathrm{~L}$; patient age ranged from 36 to 89 years, with a male predominance (4:1). In addition, this complex mutation was mainly found in Chinese NSCLC patients; only 1 case was in a different ethnicity (Italian). ${ }^{9-11}$ No strong association was observed between smoking status and the H833V/H835L double mutation.
Among all the reported cases, our case is the first to have a T790M-resistant alteration. This mutation has been detected as a "second-site mutation" in $>50 \%$ of EGFR mutations commonly observed in lung cancer that have developed acquired resistance to first-generation TKI. ${ }^{12}$ Our case showed that emergence of the T790M mutation is an important mechanism underlying the acquired resistance to second-generation TKI in patients harboring complex mutations of H833V and H835L. Previous studies have reported that the advantage of using osimertinib is that it not only inhibits sensitizing EGFR mutations but also the T790M mutant. In this study, we found that osimertinib could be a treatment option after afatinib resistance in NSCLC patients with complex mutations of $\mathrm{H} 833 \mathrm{~V}$ and $\mathrm{H} 835 \mathrm{~L}$. Our case is also the first in which the efficacy of a third-generation TKI in a patient with the H833V and H835L double mutation was evaluated. Preclinical data support the ability of osimertinib to cross the blood-brain barrier and penetrate the central nervous system (CNS), ${ }^{13}$ and as second-line treatment, osimertinib showed superior efficacy in CNS metastases compared with platinum chemotherapy. ${ }^{14,15}$ The present case also supports osimertinib as a good choice for patients with NSCLC harboring double mutations of H833V and $\mathrm{H} 835 \mathrm{~L}$ with brain metastases. Recently, Kohsaka et a ${ }^{13}$ conducted a high-throughput functional evaluation of EGFR gene variants of unknown significance in cancer and found that the L833V mutation was a gefitinib-resistant mutation. Consistent with this observation, our patient also showed primary resistance to gefitinib. In total, this subset of NSCLC harboring complex mutations of $\mathrm{H} 833 \mathrm{~V}$ and $\mathrm{H} 835 \mathrm{~L}$ may be sensitive to second- and third-generation EGFR TKIs, rather than first-generation EGFR TKIs.

Except for $\mathrm{H} 833 \mathrm{~V}$ and $\mathrm{H} 835 \mathrm{~L}$, the present case also had R670W mutation. Until now, there were no reported patients who had EGFR R670W mutation. It remains unclear about

Table I Clinical characteristics and prognosis of NSCLC patients harboring the L833V/H835L complex mutation

\begin{tabular}{|c|c|c|c|c|c|c|c|c|c|c|}
\hline & Age & Sex & Race & Smoking & Mutations & Histology & Sample & Assay & Treatment & PFS \\
\hline I & NA & Male & Asian & NA & L833V/H835L & Adenocarcinoma & Tumor tissues & Direct sequencing & NA & NA \\
\hline 2 & 76 & Female & Asian & $\mathrm{NA}$ & L833V/H835L & Adenocarcinoma & Tumor tissues & Direct sequencing & NA & NA \\
\hline 3 & 89 & Male & Asian & Light smoker & L833V/H835L & Adenocarcinoma & Tumor tissues & ARMS-PCR & $\begin{array}{l}\text { Gefitinib } \\
\text { (second-line) }\end{array}$ & 34 weeks \\
\hline 4 & 48 & Male & Asian & Nonsmoker & L833V/H835L & Adenocarcinoma & Tumor tissues & ARMS-PCR & $\mathrm{PEM}+\mathrm{C}$ & $>16$ weeks \\
\hline 5 & 70 & Male & Italian & Former smoker & L833V/H835L/E709K & Adenocarcinoma & Tumor tissues & NA & Afatinib & $>2$ months \\
\hline 6 & 36 & Male & Asian & Nonsmoker & L833V/H835L/R670W & Adenocarcinoma & Plasma cfDNA & NGS & $\begin{array}{l}\text { Afatinib } \\
\text { (third-line) } \\
\text { Osimertinib } \\
\text { (fifth-line) }\end{array}$ & $\begin{array}{l}>7 \text { months } \\
>3 \text { months }\end{array}$ \\
\hline
\end{tabular}

Abbreviations: ARMS-PCR, amplification refractory mutation system-polymerase chain reaction; cfDNA, cell-free DNA; NA, not available; NGS, next-generation sequencing; NSCLC, non-small cell lung cancer; PFS, progression-free survival; PEM + C, pemetrexed + cisplatin. 
the response of R670W mutation to EGFR TKI. Yang et $\mathrm{al}^{8}$ reported an 89-year-old Chinese male harboring a heterozygous complex mutation of L833V and H835L who showed a good response to gefitinib. However, the patient in this study, who harbored the triple L833V/H835L/R670 mutation, was resistant to gefitinib, suggesting that R670W may contribute to resistance to first-generation EGFR TKIs. However, we also observed L833V/H835L recurrence when patients had brain metastases, but lacked the R670W mutation. Thus, it is possible that the $\mathrm{L} 833 \mathrm{~V} / \mathrm{H} 835 \mathrm{~L}$ mutation is the major driving molecular event in NSCLC patients. Although it may be attributed to individual heterogeneity, the role of EGFR R670W in NSCLC patients should be explored in future studies.

One important implication of the present case study is the importance of NGS and liquid biopsy in detecting molecular alterations. If we had only performed ARMS-PCR for common EGFR mutations, the uncommon EGFR mutation H833V/H835L/R670W would not have been detected, and the patient would have missed the opportunity to be treated with TKIs, to which he had a good tolerance and favorable response. This was especially beneficial as this patient had intolerable side effects with chemotherapy. Therefore, as a high-throughput technique, NGS may allow more information to be obtained about molecular alterations, especially for the detection of uncommon mutations. Of all 6 patients reported in Table 1, cfDNA was only used in the present case. Metastatic bone specimens cannot be used for detection due to decalcification, ${ }^{16}$ and primary NSCLC lesions that are located close to the mediastinum and diaphragm cannot be obtained. Thus, liquid biopsy was the only way to detect the driving mutations in this patient, and dynamic liquid biopsy was necessary to determine the mechanism underlying the acquired resistance to TKI, which was necessary to guide clinical decisions about treatment strategy. After we detected the emergence of T790M, the patient received osimertinib and experienced partial remission.

In conclusion, because there are a relatively small number of cases with rare and/or complex mutations, it is not feasible to design a prospective trial. In addition, the impact of other rarer EGFR mutations is still unclear and remains difficult to define due to the very small number of cases. Thus, single case reports and small case series may be relevant for clinical practice orientation. Translational studies and establishment of a database will be instrumental for addressing many of these unanswered questions.

\section{Acknowledgments}

We thank LetPub for its linguistic assistance during the preparation of this manuscript. We thank the patient and her family. We also thank Qiang Chen from Burning Rock Biotech for valuable discussion and support. This study was supported by the Shanghai Sailing Program (grant number 17YF1425200, 2017); Chinese National Natural Science Funding (grant number 81702249, 2017); the Young Start-up Foundation of Changzheng Hospital (grant number 2015CZQN07, 2015); and Shanghai Municipal health and Family Planning Commission Foundation (grant number 201540174, 2015). The funder has no role in the study design, data collection and analysis, decision to publish, or preparation of the manuscript.

\section{Disclosure}

The authors report no conflicts of interest in this work.

\section{References}

1. O'Kane GM, Bradbury PA, Feld R, et al. Uncommon EGFR mutations in advanced non-small cell lung cancer. Lung Cancer. 2017;109: 137-144.

2. Kobayashi Y, Togashi Y, Yatabe Y, et al. EGFR Exon 18 mutations in lung cancer: molecular predictors of augmented sensitivity to afatinib or neratinib as compared with first- or third-generation TKIs. Clin Cancer Res. 2015;21(23):5305-5313.

3. Shi Y, Au JS, Thongprasert S, et al. A prospective, molecular epidemiology study of EGFR mutations in Asian patients with advanced non-small-cell lung cancer of adenocarcinoma histology (PIONEER). J Thorac Oncol. 2014;9(2):154-162.

4. Chiu CH, Yang CT, Shih JY, et al. Epidermal growth factor receptor tyrosine kinase inhibitor treatment response in advanced lung adenocarcinomas with G719X/L861Q/S768I mutations. J Thorac Oncol. 2015;10(5):793-799.

5. Yang JC, Sequist LV, Geater SL, et al. Clinical activity of afatinib in patients with advanced non-small-cell lung cancer harbouring uncommon EGFR mutations: a combined post-hoc analysis of LUX-Lung 2, LUX-Lung 3, and LUX-Lung 6. Lancet Oncol. 2015;16(7):830-838.

6. Galli G, Corrao G, Imbimbo M, et al. Uncommon mutations in epidermal growth factor receptor and response to first and second generation tyrosine kinase inhibitors: a case series and literature review. Lung Cancer. 2018;115:135-142.

7. Frega S, Conte P, Fassan M, Polo V, Pasello G. A triple rare E709K and L833V/H835L EGFR mutation responsive to an irreversible Pan-HER inhibitor: a case report of lung adenocarcinoma treated with afatinib. J Thorac Oncol. 2016;11(5):e63-e64.

8. Yang TY, Tsai CR, Chen KC, Hsu KH, Lee HM, Chang GC. Good response to gefitinib in a lung adenocarcinoma harboring a heterozygous complex mutation of L833V and H835L in epidermal growth factor receptor gene. J Clin Oncol. 2011;29(16):e468-e469.

9. Huang SF, Liu HP, Li LH, Lh L, et al. High frequency of epidermal growth factor receptor mutations with complex patterns in non-small cell lung cancers related to gefitinib responsiveness in Taiwan. Clin Cancer Res. 2004;10(24):8195-8203.

10. Zhuang Y, Xu J, Ma H, et al. A sequential method of epidermal growth factor receptor mutation detection reduces false negatives: a new case with doublet mutations of L833V and H835L in China. Clin Lung Cancer. 2013;14(3):295-300.

11. Lai RS, Xie L, Shen LS, Zhu CL, Qian J. Epithelial growth factor receptor (EGFR) exon double-sequencing analysis in NSClC. Zhonghua Zhong Liu Za Zhi. 2006;28(8):599-602.

12. Chen LY, Molina-Vila MA, Ruan SY, et al. Coexistence of EGFR T790M mutation and common activating mutations in pretreatment non-small cell lung cancer: a systematic review and meta-analysis. Lung Cancer. 2016;94:46-53. 
13. Kohsaka S, Nagano M, Ueno T, et al. Amethod of high-throughput functional evaluation of EGFR gene variants of unknown significance in cancer. Sci Transl Med. 2017;9(416):eaan6566.

14. Mok TS, Wu Y-L, Ahn M-J, et al. Osimertinib or platinum-pemetrexed in EGFR T790M-positive lung cancer. $N$ Engl J Med. 2017;376(7): 629-640.

15. Goss G, Tsai CM, Shepherd FA, et al. CNS response to osimertinib in patients with T790M-positive advanced NSCLC: pooled data from two Phase II trials. Ann Oncol. 2017;29(3):687-693.
16. Leighl NB, Rekhtman N, Biermann WA, et al. Molecular testing for selection of patients with lung cancer for epidermal growth factor receptor and anaplastic lymphoma kinase tyrosine kinase inhibitors: American Society of Clinical Oncology endorsement of the College of American Pathologists/International Association for the study of lung cancer/ association for molecular pathology guideline. J Clin Oncol. 2014; 32(32):3673-3679.

\section{Publish your work in this journal}

OncoTargets and Therapy is an international, peer-reviewed, open access journal focusing on the pathological basis of all cancers, potential targets for therapy and treatment protocols employed to improve the management of cancer patients. The journal also focuses on the impact of management programs and new therapeutic agents and protocols on

\section{Dovepress}

patient perspectives such as quality of life, adherence and satisfaction. The manuscript management system is completely online and includes a very quick and fair peer-review system, which is all easy to use. Visit http://www.dovepress.com/testimonials.php to read real quotes from published authors.

Submit your manuscript here: http://www.dovepress.com/oncotargets-and-therapy-journal 